\title{
Power efficiency of the active boundary layer control around the hump by a slotted synthetic jet generator
}

\author{
Petr Pick ${ }^{1, a}$ \\ ${ }^{1}$ Aerospace Research and Test Establishment, Engine department, Beranových 130, Prague, Czech Republic
}

\begin{abstract}
The present contribution summarizes the power efficiency of the active flow control of the boundary layer of air around a hump. The synthetic jet generator with a rectangular output part, i.e. a slot, is actuated using a modulated signal. The actuation of the synthetic jet is carried out by modulating the input voltage of acoustic transducers of the generator. This causes the decrease of the loss coefficient and the change of the mixing size area (e.g. wake). A comparison of three types of modulating signals and their influence on the loss coefficient is performed. The main advantages of modulated signal are then described.
\end{abstract}

\section{Introduction}

The synthetic jet boundary layer control is an effective method that uses an unsteady blowing of the fluid flow into the boundary layer and its periodic suction from the close surroundings. In this article, a synthetic jet generator with slot orifice is used to control the boundary layer and the wake behind two models. The velocity vector of the fluid blowing from the synthetic jet generator is perpendicular to the free stream. The small energy consumption of this setup is further mentioned. A brief description of the design stages of the efficient synthetic generator is provided. The design stages are subsequently mentioned which are applicable for manufacturing the synthetic jet generator. This generator was used to control the boundary layer and the wake as becomes evident from experimental results given. The experimental results introduced in this contribution present inherent properties of three types of modulated actuation signal. The advantages and disadvantages of these cases are considered on criteria based on energy efficiency, acoustic noise influence and the duty cycle.

\section{Synthetic jet generator cell}

In both models, a synthetic jet generator (SJG) was presented, which consisted of synthetic jet cells. Each of them was made of two acoustic transducers mounted against each other. The transducers moved in phase against each other and sucked fluid from the free surroundings into the cavity and then blew it out through a slot perpendicular to the free stream flow.

The cell design was performed according to [1] and the cavity of the cell was optimized by [2]. In accordance with [3] all the work regimes are present in the area of synthetic jet existence, see

Figure 1. The necessary experimental verification of the frequency response characteristic is performed in [4]. The output parameters are described in Table 1. The nondimensional frequency is defined according to equation (2.1), the momentum coefficient is defined by equation (2.2), the maximal velocity of synthetic jet flow is marked $U_{\text {jmax }}$. In these equations, the variable $f$ means frequency, the value of which is dependent on what case of actuation is used, i.e. whether a carrying or a modulating frequency. $X_{t e}$ represents the length of the SJG affected area. In both models it was $0.2 \mathrm{~m}), U_{\infty}$ is the free stream velocity; $h$ means slot width and $v$ means kinematic viscosity. Variable $c$ represents the length of the hump from the leading edge to the trailing edge and it is equal to $0.395 \mathrm{~m}$. The carrying frequency is marked $f_{C}$ and the modulating frequency is marked $f_{M}$.

Table 1 The synthetic jet cell performance

\begin{tabular}{|c|c|c|c|}
\hline & $\mathrm{u}_{\text {imax }}(\mathrm{m} / \mathrm{s})$ & $\mathrm{F}^{+}(-)$ & $\mathrm{c}_{\mu}(\%)$ \\
\hline harmonic signal & 32.5 & $0.3-6.16$ & 1.04 \\
\hline modulated signal & 41.5 & $0.3-3$ & 1.7 \\
\hline
\end{tabular}

$$
\begin{gathered}
F^{+}=\frac{f \cdot X_{t e}}{U_{\infty}} . \\
c_{\mu}=\frac{h}{c}\left(\frac{U_{j \max }}{U_{\infty}}\right)^{2} . \\
\operatorname{Re}=\frac{U_{\infty} \cdot c}{v} .
\end{gathered}
$$

\footnotetext{
${ }^{\mathrm{a}}$ Corresponding author: petr.pick@seznam.cz
} 


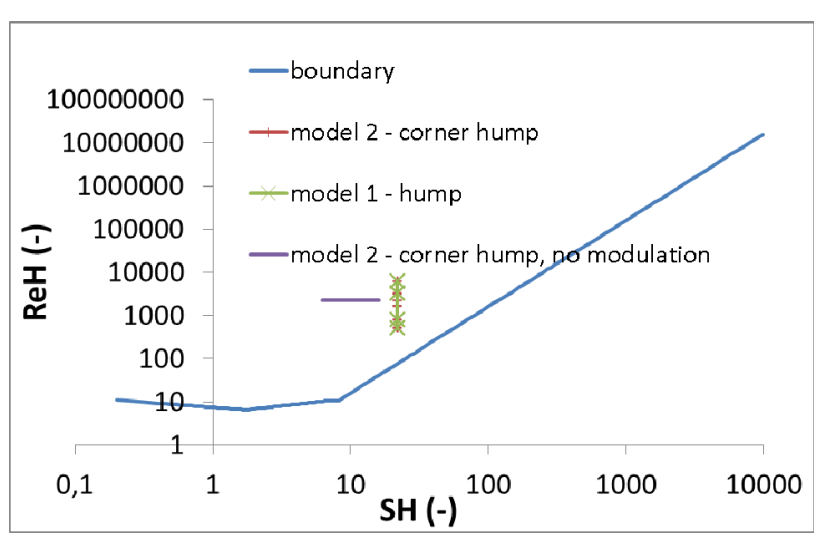

Figure 1. Working area of the used synthetic jet generators according to used model configuration of the measurement.

\section{Actuating signal}

The cells in the modules were connected in two parallel branches so that the neighbouring cells would be actuated with different phase shifts. Both synthetic jet modules were actuated with frequency $f_{C}=370 \mathrm{~Hz}$, which is both the carrying actuation frequency and the resonance cavity frequency of each cell. The output slot of the SJG, which was one millimetre wide, was placed in the location of maximum thickness of both models.

The parallel circuit wiring of the cells makes it possible to use the phase shift of the modulating or carrying signal.

Four types of actuating signal were used. Its parameters are described in Table 2.

Table 2 Cases of actuating signal

\begin{tabular}{|c|c|c|c|c|}
\hline & $\mathrm{f}_{\mathrm{C}}(\mathrm{Hz})$ & $\mathrm{f}_{\mathrm{M}}(\mathrm{Hz})$ & $\begin{array}{c}\text { phase shift } \\
\text { of } \mathrm{f}_{\mathrm{C}}\end{array}$ & $\begin{array}{c}\text { phase shift } \\
\text { of } \mathrm{f}_{\mathrm{M}}\end{array}$ \\
\hline Case A & $10-370$ & ----- & ----- & ----- \\
\hline Case B & 370 & $10-90$ & $0^{\circ}$ & $0^{\circ}$ \\
\hline Case C & 370 & $10-90$ & $0^{\circ}$ & $180^{\circ}$ \\
\hline Case D & 370 & $10-90$ & $180^{\circ}$ & $0^{\circ}$ \\
\hline
\end{tabular}

\section{Experimental setup}

The aerodynamic tunnel depicted in Figure 2 had an open cycle. The closed test section was $300 \mathrm{~mm}$ wide and $200 \mathrm{~mm}$ high. The turbulence intensity on the entrance of the test section was $1.3 \%$ and the momentum thickness $8 \mathrm{~mm}$ at $\mathrm{Re}=160,000-250,000$. Reynolds number is defined in equation (2.3). The working medium in the aerodynamic tunnel was air. The test section and measurement plane are shown in Figure 5.

Two models were used for testing. The one is a hump of a prismatic shape, which was based on ERCOFTAC ${ }^{b}$ geometric data (see keyword "hump"). It was placed horizontally on the bottom of the wind tunnel test section to serve as the body to be flown around. The model dimensions are $300 \times 400 \times 50 \mathrm{~mm}$.

The other model is called a corner hump and is assembled using the first horizontal module and a second vertical module of the hump. It was placed on the side wall of the aerodynamic tunnel. The corner hump forms a big corner vortex of the secondary flow. The model dimensions are $300 \times 400 \times 200 \mathrm{~mm}$.

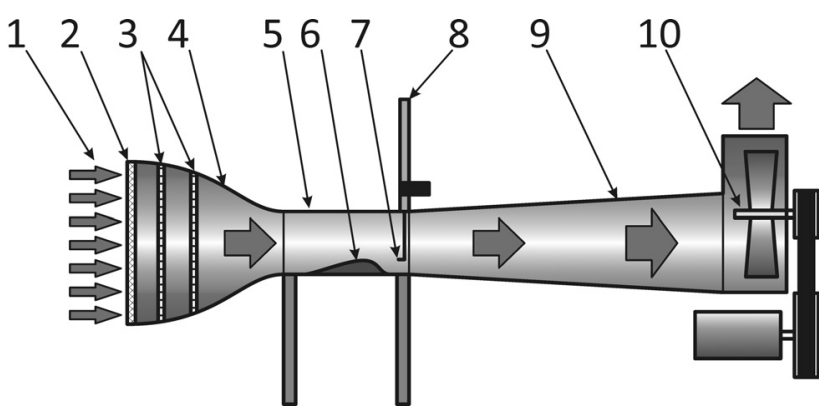

Figure 2. Schema of the open-circuit wind tunnel with a closed test section, 1 - air intake; 2 - dust filter; 3 - settling chamber; 4 - contraction cone; 5 - test section; 6 - model; 7 - probe holder; 8 - traversing unit; 9 - diffuser; 10 -drive section.

The SJG placed in hump consisted of nine active cells. The SJG placed in the horizontal module of the corner hump consisted of eight active cells and the SJG placed in the vertical module of the corner hump of five such cells. Connection diagram for hump can be seen on the Figure 3, where red and blue line represents two parallel branches of electric circuit. These two branches are connected to amplifier which is controlled by signal generator. On the Figure 4 is wiring diagram for corner hump. On these figures is described configuration of SJG cells in corner which blowing air alternatively.

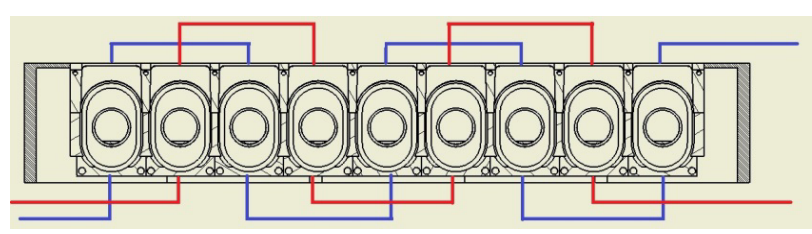

Figure 3. Wiring diagram of SJG in hump, two parallel branches are marked.

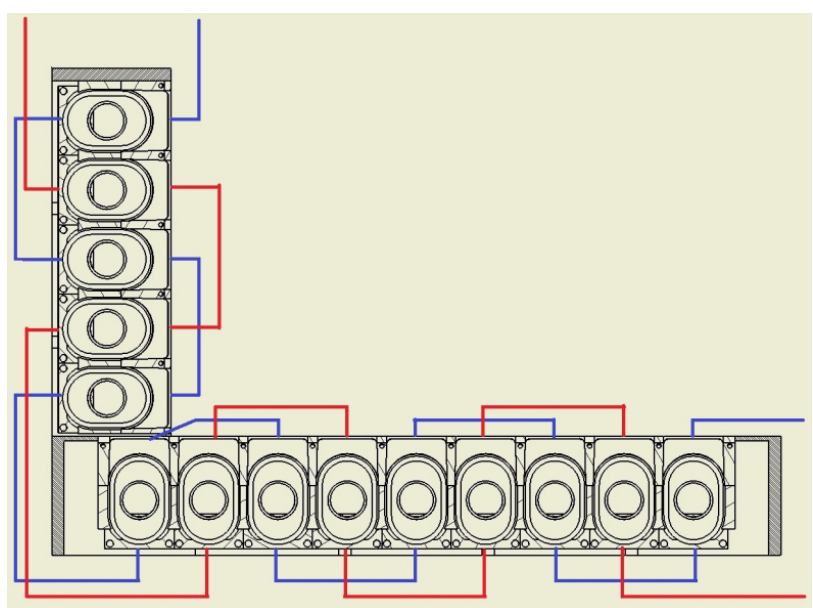

Figure 4. Wiring diagram of SJG in corner hump, two parallel branches are marked.

${ }^{\mathrm{b}}$ http://www.ercoftac.org/ 
The total pressure was calculated from the data measured by the five-hole probe placed in the plane of $530 \mathrm{~mm}$ from the leading edge of the hump.
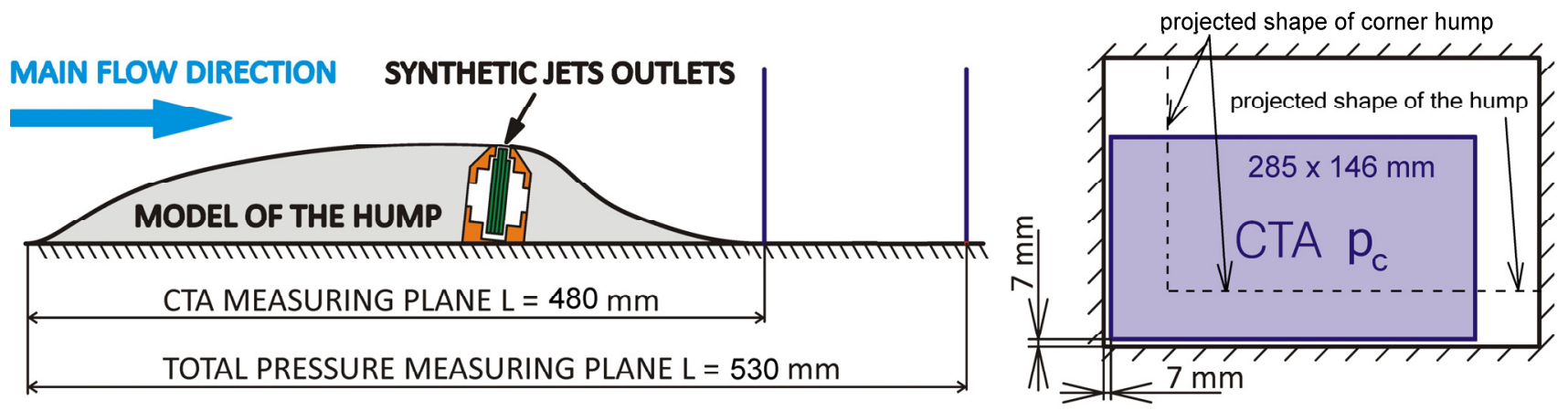

Figure 5. The section of the hump shape with synthetic jet (fore-and-aft section), depicting the corner hump and the hump projection into measurement planes.

The velocity of the flow, measured with HWA probe by CTA method, was measured in the plane $480 \mathrm{~mm}$ from the leading edge of the hump. The dimensions of the area measured by the CTA probe and five-hole probe were $285 \mathrm{~mm} \mathrm{x} 145 \mathrm{~mm}$. The starting points of these measurement areas were situated $7 \mathrm{~mm}$ from both sides of the left bottom corner of the wind tunnel test section. The measurement area was not symmetrical; it was shifted to bottom wall and the left side wall, as viewed from the free stream. Therefore, the wall effect of the synthetic jet is slightly visible on the right side of the wind tunnel.

The noise measurement was made with TASCAM handheld recorder DR02. This recorder was placed in front of the intake of the wind tunnel four meters upwind from synthetic jet in axis of the wind tunnel. This location makes it possible to have a direct view of the synthetic jet; there are only a dust filter and a settling chamber in-between. A built-in microphone with a frequency response of $20 \mathrm{~Hz}-22 \mathrm{kHz}+1 /-3 \mathrm{~dB}$ was used to record the sound, the recording parameters of the WAV sound file being $96 \mathrm{kHz} / 24$-bit. To compare the recorded sounds an average RMS power was used.

\section{Post-processing of measured data}

The partial loss coefficient was defined in equation (5.1).

$$
d \xi_{\text {tot }}=\frac{\left.p_{c \operatorname{Re} f}-p_{c} \text { (probe }\right)}{p_{d \operatorname{Re} f}} .
$$

In this equation, the $p_{c \operatorname{Re} f}$ is the reference total pressure in the wind tunnel before the hump, $p_{c}$ (probe) is the total pressure measured by the five-hole probe, $p_{d \operatorname{Re} f}$ is the reference dynamic pressure before the hump. Total loss coefficient is defined in equation (5.2).

$$
\xi_{\text {tot }}=\frac{\sum d \xi_{\text {tot }} \cdot d A_{\text {grid }}}{A_{\text {grid }}} .
$$

In this equation, the $\xi_{\text {tot }}$ is the loss coefficient, $d \xi_{\text {tot }}$ is the partial loss coefficient, $d A_{\text {grid }}$ is the partial area of the grid in which the loss coefficient is measured, and $A_{\text {grid }}$ is the total measuring area.

The average loss energy $w_{\text {loss }}$ is expressed by equation (5.3). The saved energy is then the difference of the total loss coefficients for cases with no active boundary layer control and with one, as expressed by equation (5.4). The final added specific energy is equal to the kinetic energy added to the fluid flow by a synthetic jet; see equation (5.5).

$$
\begin{gathered}
w_{\text {loss }}=\xi_{\text {tot }}(0) \cdot \frac{1}{2} U_{\infty}^{2} . \\
w_{s}=\left[\xi_{\text {tot }}(0)-\xi_{\text {tot }}(f)\right] \frac{1}{2} U_{\infty}^{2} . \\
w_{\text {add }}=\frac{P_{k i n}}{\dot{m}} .
\end{gathered}
$$

In order to compare the modulated and nonmodulated actuating signal the value of the electric power of SJG is substituted for variable $P_{k i n}$. To compare the measurements of the loss coefficient the description "loss coefficient decrease" is used in figures. It expresses the way the total loss coefficient is related to the basic value measured with zero actuation influence.

If the amount of energy that was saved (5.4) is negative, it means that the influence of boundary layer control leads to an increase in drag against the basic case without active flow control.

Analogically to that, if the amount of energy that was saved is positive, the influence of the boundary layer control results in a decrease in drag value of the loss coefficient against the basic case without active flow control.

Further, the effectiveness of boundary layer control can be expressed as the difference between the saved energy $w_{s}$ and the added energy $w_{\text {add }}$ standardized to the spent energy during the flowing around the model with zero active flow control; see equation (5.6).

$$
\eta_{E J}=\frac{w_{s}-w_{a d d}}{w_{\text {loss }}} \text {. }
$$

A positive value of the effectiveness reflects the positive influence of the boundary layer control through energy saving. Again in analogy, a negative value means 
that more energy was spent than is the case when there is zero active control, i.e. the basic case.

To compare the values in the figures, the description "actuation efficiency of kin. energy" is used. The actuation efficiency is marked this way in relation to the kinetic energy added to the fluid flow. In tables, the terms "kin.energy" and "el.energy" are used. It marks the actuation efficiency in relation to the kinetic or electric energy that was added.

During measurements using the HWA probe by the CTA method the velocity in traversing area is measured. From these measured data the mean velocity (5.7) and the turbulence intensity (5.8) are calculated.

$$
\begin{aligned}
u_{R M S} & =\sqrt{\overline{u^{\prime}(t)^{2}}} . \\
T u & =\frac{u_{R M S}}{\bar{u}} .
\end{aligned}
$$

In this formula $u^{\prime}(t)$ is the fluctuation part of the flow velocity measured by hot wire probe (CTA method), $\mathrm{u}_{\mathrm{RMS}}$ is the mean flow velocity and $\bar{u}$ is the average flow velocity calculated from the whole sampling period of a certain measurement point. The sampling frequency of the HWA probe was $30 \mathrm{kHz}$, the sampling period was two seconds.

\section{Comparison of the power efficiency of the SJG cell}

According to [4], a mathematical model of the synthetic jet was provided, in which the synthetic jet is blown by the generator.

The model takes the following into consideration: the output jet velocity, the kinetic energy added per time unit and the electric power into the generator. The kinetic energy is expressed by (6.1) and the electric power by (6.2).

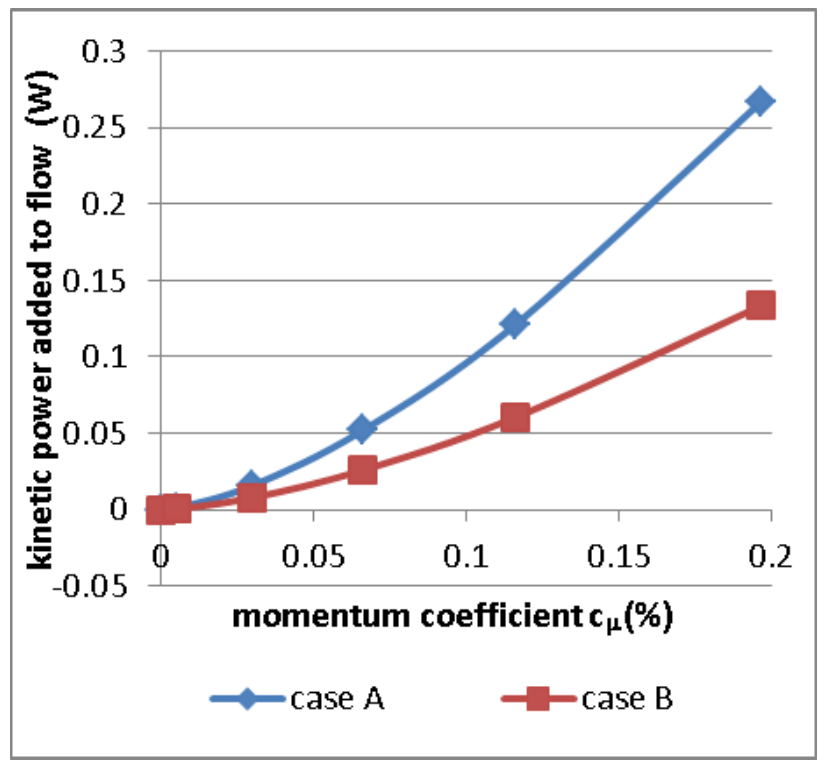

Figure 6. A comparison of kinetic power added to flow for two cases of actuation.

$$
\begin{gathered}
P_{k i n}=\frac{1}{2} m_{j} \cdot u_{j}^{2} \cdot T_{\text {duty }} . \\
P_{e l}=\frac{R I_{\max }^{2}}{2} .
\end{gathered}
$$

In these equations, $m$ is the mass flow through the SJG orifice, $u_{j}$ the average the output jet velocity from the orifice, $T_{\text {duty }}$ is the duty cycle, $R$ is the electric resistance of acoustic transducer and $I_{\max }$ is the electric current.

A comparison of the kinetic power added to the stream for the Case A signal and the Case B signal can be seen in Figure 6. Figure 7 shows the input power for the same cases.

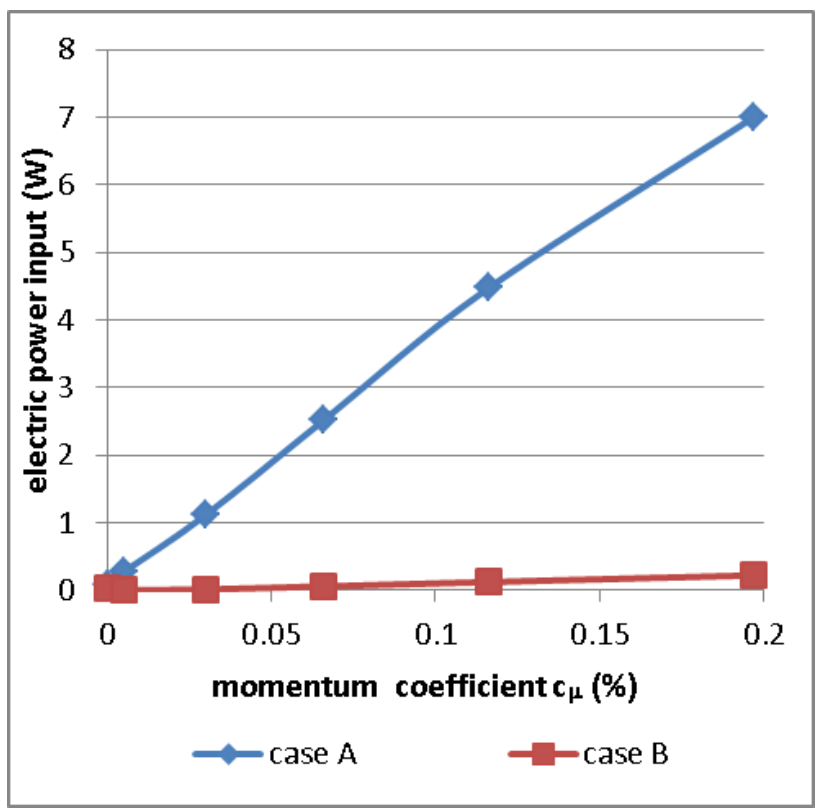

Figure 7. Comparison of required electric power input for producing of same momentum coefficient for two cases of actuation.

\section{Measurement results}

The measurement of actuation efficiency was performed on the hump at a free stream velocity of $12 \mathrm{~m} / \mathrm{s}$ in the inlet of the test section. The non-dimensional frequency for this setup was $F^{+}=1.2$ which also corresponds to the maximal sensitivity of the boundary layer on the modulation frequency $f_{M}=72 \mathrm{~Hz}$. This frequency was obtained by measuring the decrease of the loss coefficient of the flow around the hump [5]. Figure 8 shows the relation between the actuating efficiency of the added kinetic energy the free stream and momentum coefficient $c_{\mu}$ for two cases of actuating signal. The measured values of the actuation efficiency of the kinetic and the electric energy are described in

Table 3.

Concerning the corner hump, the measurement of the actuation efficiency was performed. It was the efficiency of the added kinetic energy for two free stream velocity regimes, 8 and $12 \mathrm{~m} / \mathrm{s}$. Reynolds number relating to these two regimes depended on the characteristic dimension, which were described by equation (2.3) for $c=0.395 \mathrm{~m}$ 
$R e_{c}=208,700$ or $R e_{c}=312,400$. Alternatively, the test section of this tunnel can be viewed as a channel flow, hence the characteristic dimension can be the hydraulic diameter $D_{H}=0.24 \mathrm{~m}$ and $R e_{D h}=140890$ or $R e_{D h}=$ 210880. The one velocity regime with three cases of actuation signal is shown in Figure 9.

The other velocity regime with four cases of actuation signal is shown in Figure 10 and the plotted values of efficiency with the added kinetic and electric energy are set in

Table 4. This table makes it possible to compare the added kinetic energy and the electric energy in terms of the average energy loss (5.3). It is evident that there is a small difference between added kinetic energy and electric energy needed by SJG in relation to average loss energy. This difference depends on section 0 results.

An influence of the duty cycle was observed too. Three values of the duty cycle were used, 30,50 and $70 \%$ for three cases of actuation. They are shown in Figure 11.
In all experiments, except for this measurement, a duty cycle of $50 \%$ was used.

For the last mentioned data set, the turbulence intensity was plotted in Figure 13 and Figure 14 for Case C and Case D respectively. The momentum coefficient was set to $c_{\mu}=0.89 \%$. The marked places in figures clearly show areas of changes in turbulence intensity. In Case C, actuation cause the strong decrease of turbulence intensity in lower region of measurement area. The effect of the phase shift of carrying or modulation frequency on the corner vortex is not so strong.

Further, the acoustic noise of the synthetic jet was measured, as can be seen in Figure 12 and Figure 15 for a free stream velocity of 8 or $12 \mathrm{~m} / \mathrm{s}$. The level $0 \mathrm{~dB}$ represents the condition of a wind tunnel that was turned off, the values of $c_{\mu}=0$ are equal to the noise produced by the wind tunnel running on a velocity regime setting. Case A is not clearly visible because the noise produced in this case is close to the base noise of wind tunnel.

Table 3 Measured values of actuating efficiency of the added kinetic energy and the electric input for hump at $12 \mathrm{~m} / \mathrm{s}$

\begin{tabular}{|c|c|c|c|c|c|c|c|c|}
\hline & $c_{\mu}(\%)$ & 0.015 & 0.035 & 0.08 & 0.142 & 0.222 & 0.395 & 0.618 \\
\hline \multirow{3}{*}{ Case B } & Kin. energy & $8.69 \%$ & $7.20 \%$ & $5.29 \%$ & $4.24 \%$ & $4.50 \%$ & $-1.23 \%$ & $-3.15 \%$ \\
\hline & El. energy & $8.69 \%$ & $7.15 \%$ & $5.03 \%$ & $3.76 \%$ & $3.93 \%$ & $-1.91 \%$ & $-4.16 \%$ \\
\hline & $c_{\mu}(\%)$ & 0.025 & 0.099 & 0.223 & 0.396 & 0.618 & & \\
\hline \multirow[b]{2}{*}{ Case C } & Kin. energy & $4.49 \%$ & $1.84 \%$ & $2.14 \%$ & $-1.48 \%$ & $-5.06 \%$ & & \\
\hline & El. energy & $4.48 \%$ & $1.38 \%$ & $1.43 \%$ & $-2.31 \%$ & $-6.29 \%$ & & \\
\hline
\end{tabular}

Table 4 Measured values of actuating efficiency of the added kinetic energy and the electric input for a corner hump at $12 \mathrm{~m} / \mathrm{s}$

\begin{tabular}{|c|c|c|c|c|c|c|c|c|c|}
\hline & $c_{\mu}[\%]$ & 0.016 & 0.036 & 0.063 & 0.099 & 0.223 & 0.396 & 0.618 & 0.89 \\
\hline \multirow{3}{*}{ Case B } & Kin. energie & $3.14 \%$ & $2.83 \%$ & $2.47 \%$ & $2.15 \%$ & $1.39 \%$ & $0.76 \%$ & $0.33 \%$ & $-0.23 \%$ \\
\hline & El. energy & $3.14 \%$ & $2.83 \%$ & $2.47 \%$ & $2.14 \%$ & $1.39 \%$ & $0.75 \%$ & $0.32 \%$ & $-0.24 \%$ \\
\hline & $\mathrm{c}_{\mu}[\%]$ & 0.016 & 0.036 & 0.099 & 0.222 & 0.396 & 0.618 & 0.89 & \\
\hline \multirow{3}{*}{ Case C } & Kin. energie & $3.20 \%$ & $2.59 \%$ & $2.45 \%$ & $1.90 \%$ & $1.85 \%$ & $1.87 \%$ & $1.77 \%$ & \\
\hline & El. energy & $3.20 \%$ & $2.59 \%$ & $2.45 \%$ & $1.90 \%$ & $1.84 \%$ & $1.87 \%$ & $1.75 \%$ & \\
\hline & $c_{\mu}[\%]$ & 0.016 & 0.036 & 0.223 & 0.396 & 0.618 & 0.89 & & \\
\hline \multirow{3}{*}{ Case D } & Kin. energy & $1.42 \%$ & $1.87 \%$ & $0.43 \%$ & $-0.12 \%$ & $-0.22 \%$ & $-0.32 \%$ & & \\
\hline & El. energy & $1.42 \%$ & $1.87 \%$ & $0.43 \%$ & $-0.12 \%$ & $-0.23 \%$ & $-0.33 \%$ & & \\
\hline & $c_{\mu}[\%]$ & 0.0156 & 0.036 & 0.099 & 0.223 & 0.396 & 0.618 & 0.89 & \\
\hline \multirow{3}{*}{ Case B } & Kin. energy & $1.93 \%$ & $1.75 \%$ & $1.53 \%$ & $0.54 \%$ & $0.19 \%$ & $-0.29 \%$ & $-1.25 \%$ & \\
\hline & El. energy & $1.93 \%$ & $1.75 \%$ & $1.53 \%$ & $0.54 \%$ & $0.19 \%$ & $-0.29 \%$ & $-1.26 \%$ & \\
\hline & $c_{\mu}[\%]$ & 0.0008 & 0.0016 & 0.005 & 0.012 & 0.026 & 0.054 & & \\
\hline \multirow[b]{2}{*}{ Case A } & Kin. energy & $0.02 \%$ & $0.69 \%$ & $0.92 \%$ & $1.46 \%$ & $1.64 \%$ & $1.43 \%$ & & \\
\hline & El. energy & $0.02 \%$ & $0.69 \%$ & $0.91 \%$ & $1.45 \%$ & $1.64 \%$ & $1.42 \%$ & & \\
\hline
\end{tabular}




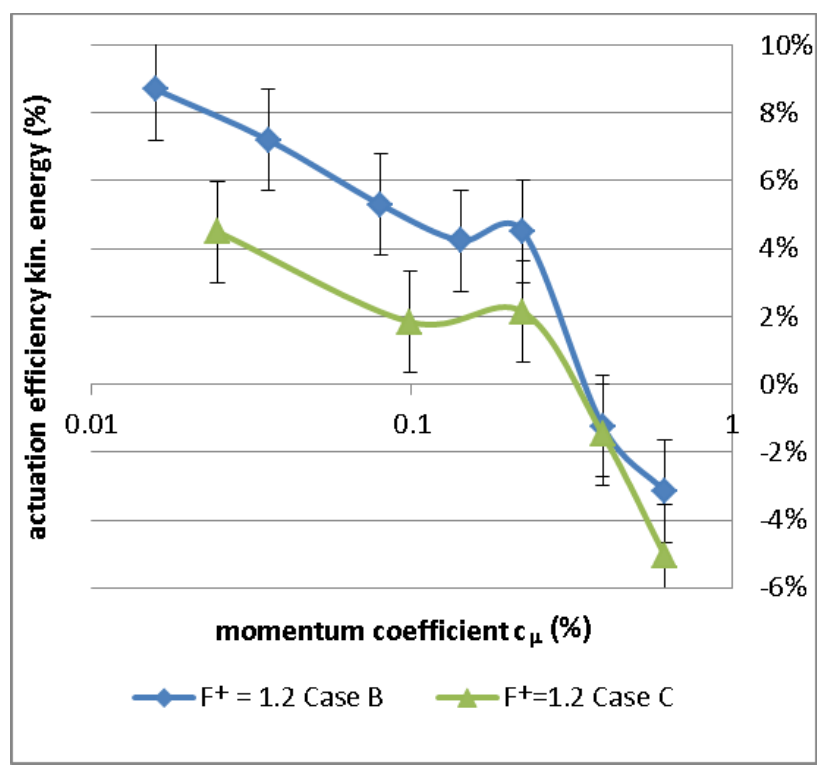

Figure 8. Actuation efficiency of kinetic energy added to the flow for the flow around the hump at a free stream velocity of $12 \mathrm{~m} / \mathrm{s}$.

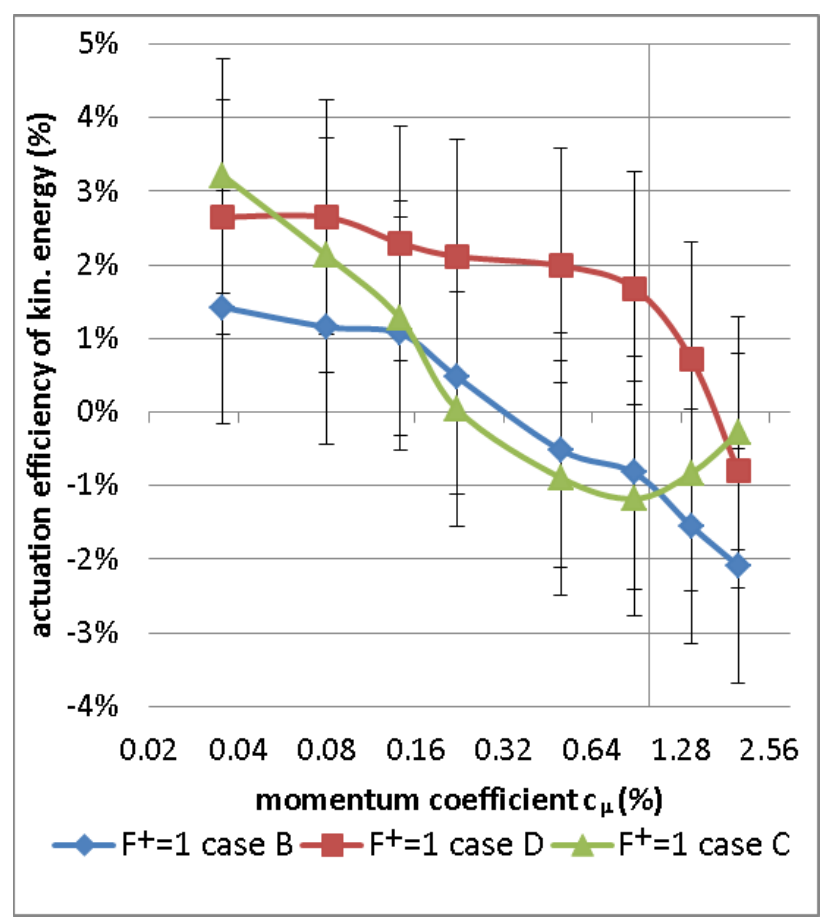

Figure 9. The actuation efficiency of added kinetic energy to flow for flow around corner hump at free stream velocity $8 \mathrm{~m} / \mathrm{s}$, corner hump.

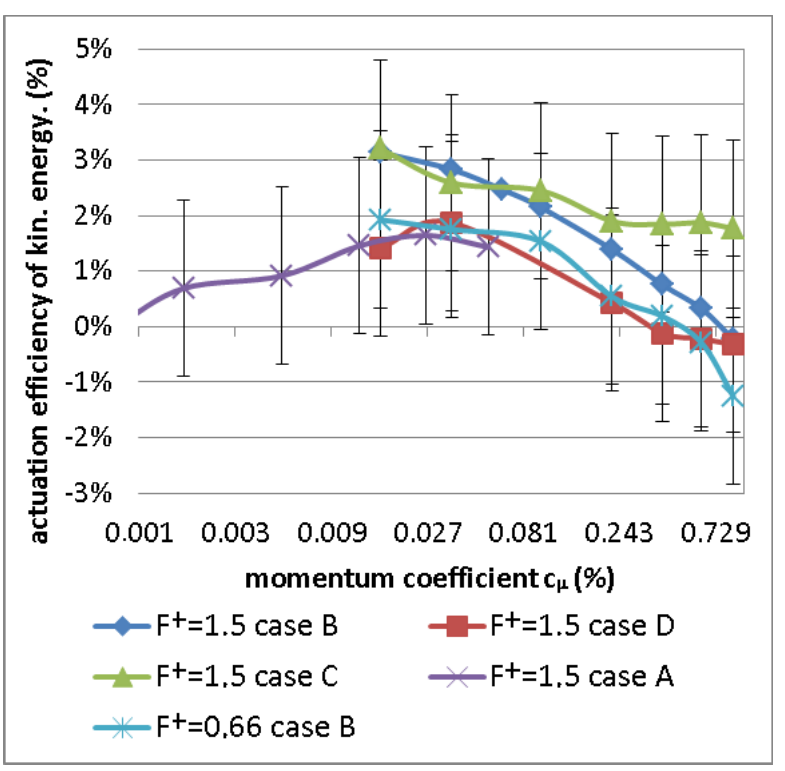

Figure 10. The actuation efficiency of added kinetic energy to the flow for the flow around the corner hump at a free stream velocity of $12 \mathrm{~m} / \mathrm{s}$, corner hump.

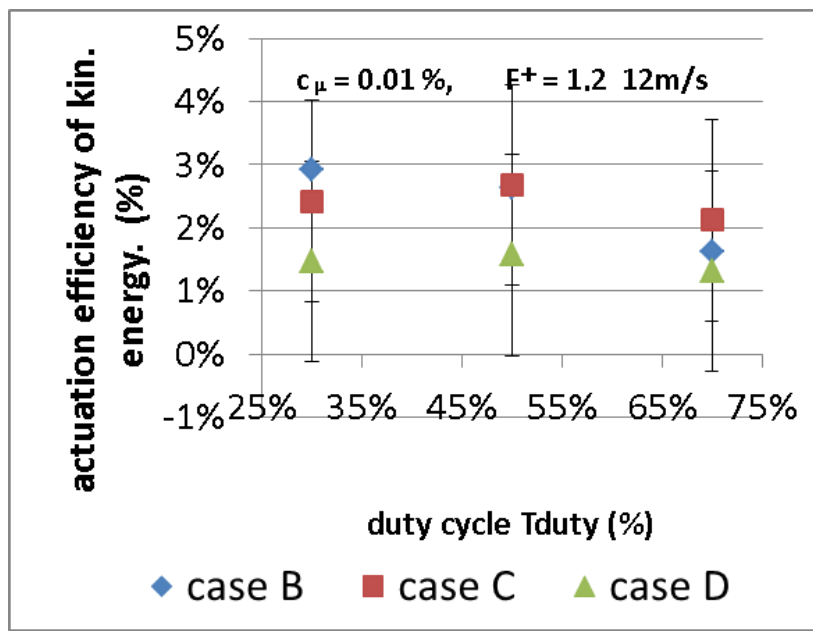

Figure 11. Influence of the duty cycle on the actuation efficiency, the corner hump.

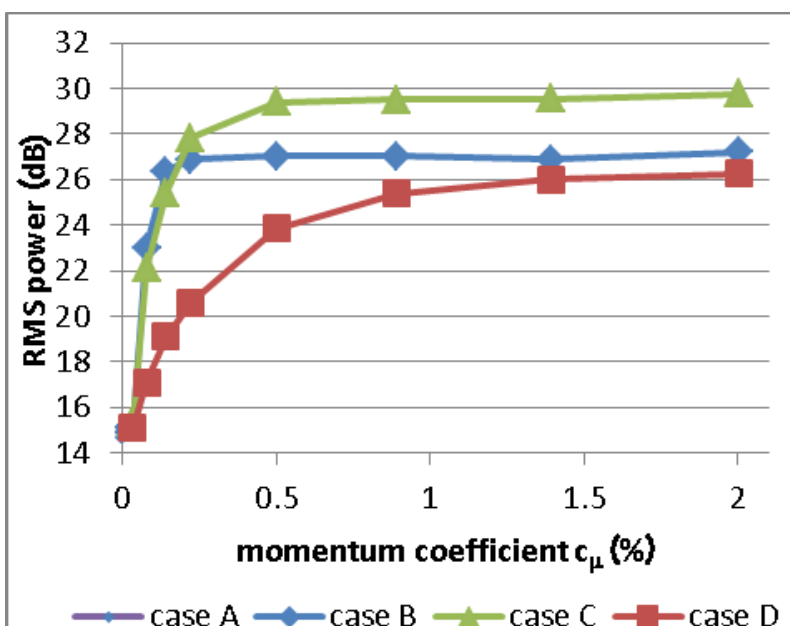

Figure 12. Acoustic noise produced by a synthetic jet related to the case of actuation signal in dependency on the momentum coefficient at a free stream velocity of $8 \mathrm{~m} / \mathrm{s}$, corner hump. 


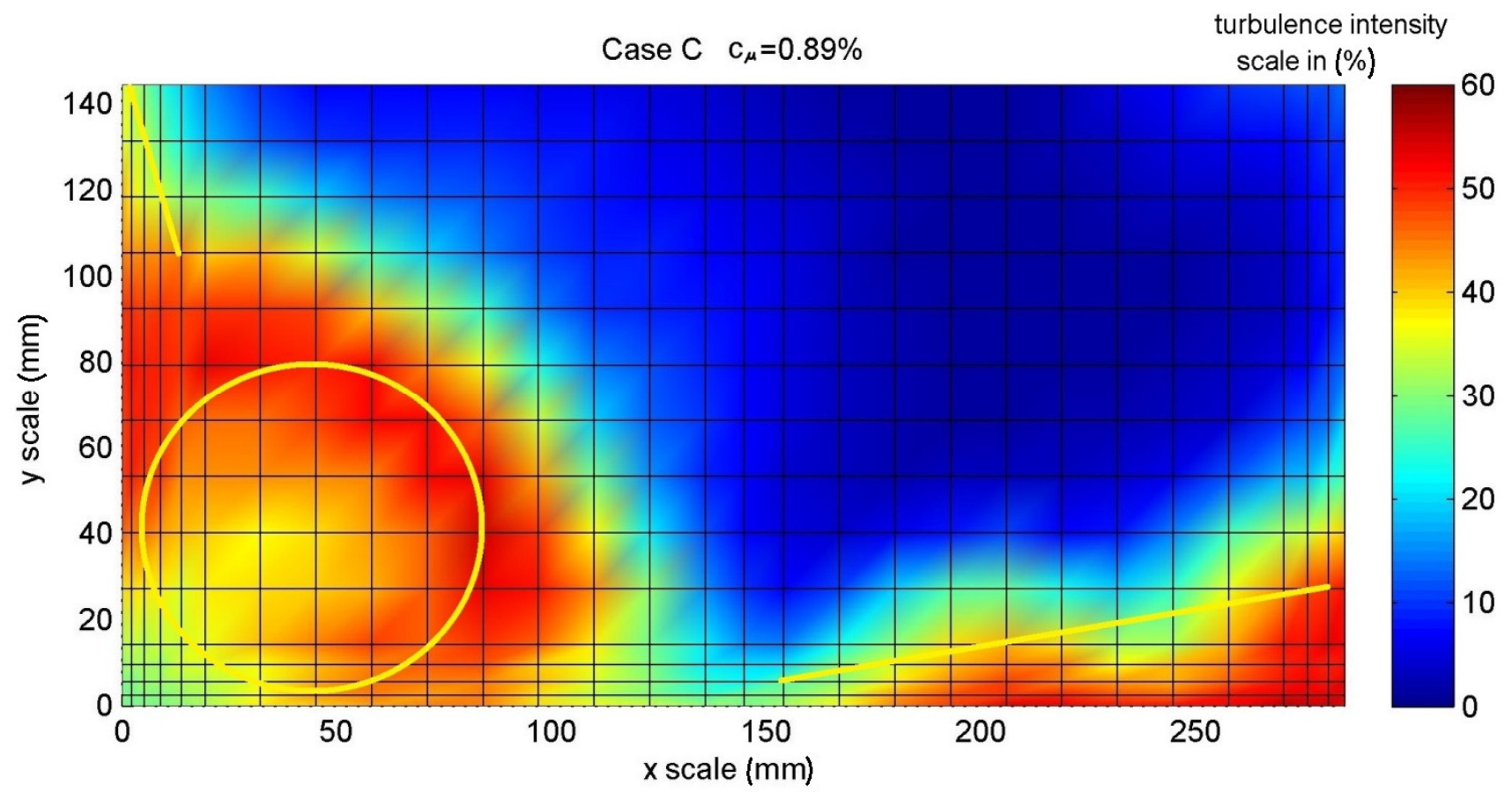

Figure 13. Turbulence intensity for Case $C$ on the corner hump at $12 \mathrm{~m} / \mathrm{s}$.

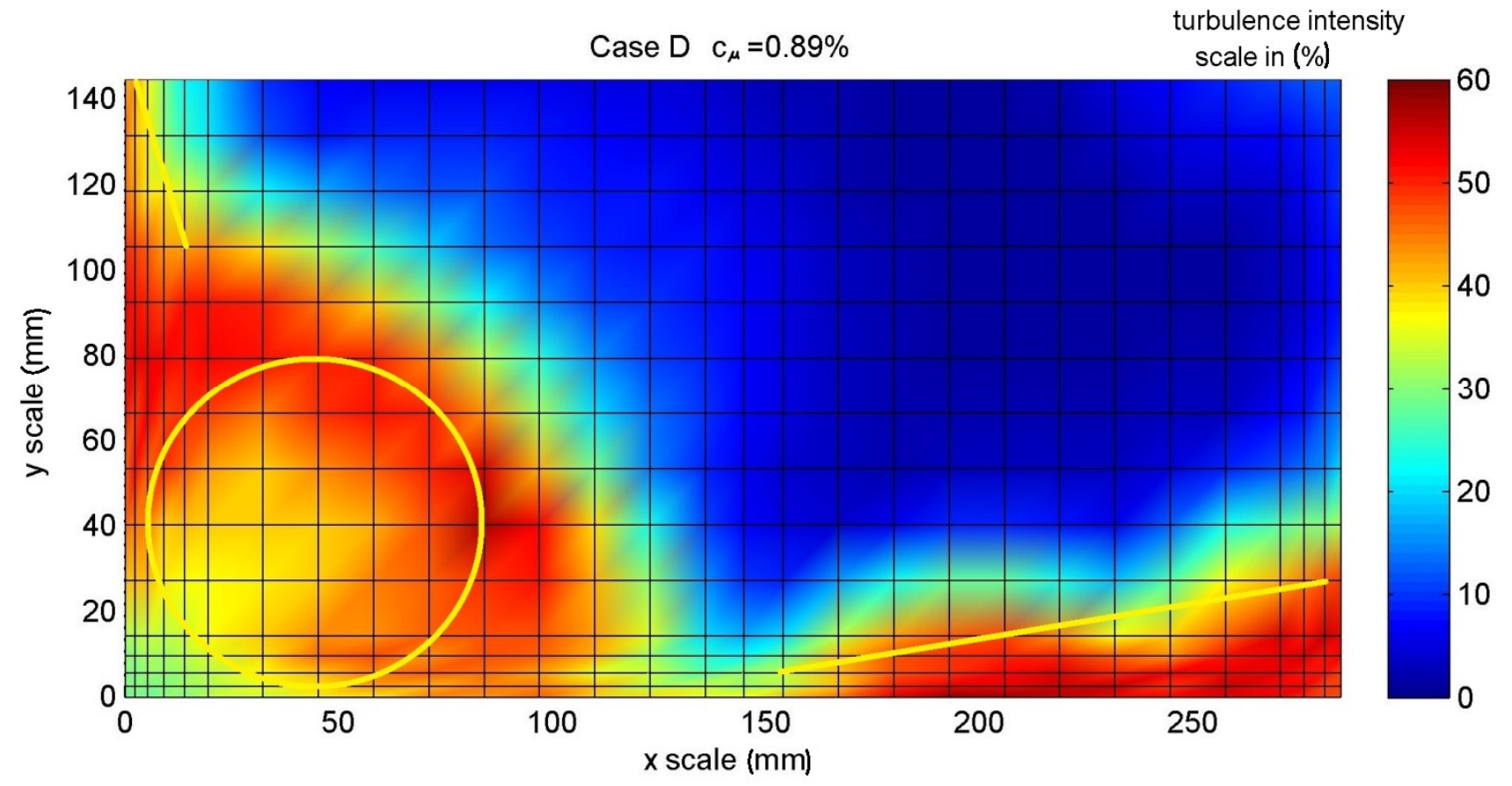

Figure 14. Turbulence intensity for Case D on the corner hump at $12 \mathrm{~m} / \mathrm{s}$. 


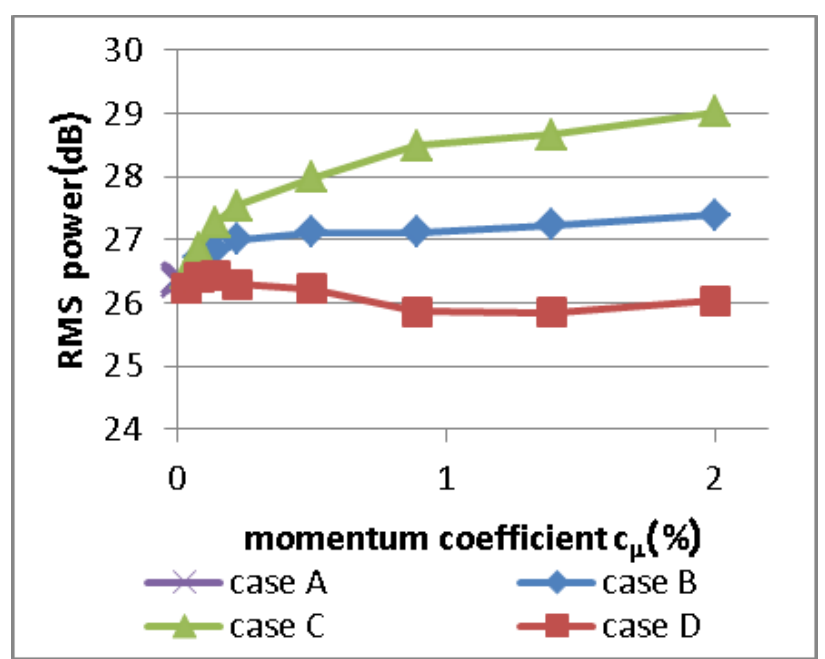

Figure 15. Acoustic noise produced by a synthetic jet related to the case of actuation signal in dependency on the momentum coefficient at a free stream velocity of $12 \mathrm{~m} / \mathrm{s}$, corner hump.

\section{Discussion}

The actuation efficiency reached $8.69 \%$ on the hump for the Case B actuation type and $4.49 \%$ for the Case C actuation type. These values were achieved at a momentum coefficient of $c_{\mu}=0.015 \%$ and $0.025 \%$, respectively. For higher values of momentum coefficient the efficiency decreases and becomes negative at $c_{\mu}=$ $0.32 \%$ and $0.3 \%$, respectively, which occurs in situations in which the synthetic jet is too strong and makes the boundary layer thicker.

As far as the corner hump is concerned, for both velocity regimes, the achieved efficiency was similar in the actuating cases $\mathrm{B}$ and $\mathrm{C}$, i.e. $3.14 \%$ and $3.21 \%$, respectively. These values were achieved for the momentum coefficient $c_{\mu}=0.035 \%$ and $0.016 \%$, respectively, at a free stream velocity of $8 \mathrm{~m} / \mathrm{s}$ and $12 \mathrm{~m} / \mathrm{s}$, respectively. In both cases, as the momentum coefficient increases, the actuation efficiency decreases. For Case $\mathrm{C}$ of the actuation signal, for higher values above $c_{\mu}=0.22 \%$ and $c_{\mu}=0.35 \%$, respectively, the amount of decrease changes. The actuation efficiency decreases very slowly for Case D at $8 \mathrm{~m} / \mathrm{s}$. This change can be related to the corner vortex behaviour. The maximal actuation efficiency at a free stream velocity of $12 \mathrm{~m} / \mathrm{s}$ was $1.87 \%$ at $c_{\mu}=0.036 \%$. Case A can only be seen in Figure 10. An increase in the actuation efficiency is evident, with the highest value $1.64 \%$ for $c_{\mu}=0.026 \%$. However, this value is reached with sixteen-time higher consumption than with the modulated actuation signal. Further, an influence of the case of actuation on the turbulence intensity was discovered. No stronger influence of actuation on the corner vortex was found at this experimental setup.

The duty cycles are able to increase and decrease the amount of energy added into the free stream. Figure 11 clearly shows that $\mathrm{T}_{\text {duty }}$ which is equal to $50 \%$ is to be recommended.

The acoustic influence of modulated actuation signal is clear from Figure 12 and Figure 15. Case D is the most silent signal for both velocities and Case $\mathrm{C}$ is the noisiest. The phase shift contributes to the decrease of noise.

\section{Conclusion}

In conclusion, six findings are evident. First it should be highlighted that at the design level, connecting the synthetic jet cells into two parallel branches helps to suppress the noise on the condition that the phase shift of the carrying frequency $f_{C}$ is applied. Second, optimizing the synthetic jet generator, with the aim of taking advantage of the Helmholtz resonant frequency of the cavity of the SJG, helps to increase the efficiency of actuation. Also, a very good efficiency of actuation was achieved at lower values of the momentum coefficient, approximately $c_{\mu}=0.03 \%$. This lower value means low energy consumption, which can further be decreased by modulating the actuation signal.

Further, the modulation signals have advantages over a harmonic signal. They cause greater decrease of losses; they can be more silent and more energy efficient. In addition, they can be quickly switched to a harmonic signal above the frequency $2 f_{M} \geq \mathrm{f}_{\mathrm{C}}$, or can use the other resonant frequency - the frequency of an acoustic transducer. A detailed description of these findings can be found in [5].

\section{Acknowledgments}

The work has been supported by the Czech Science Foundation under grants No. GA 101/08/1112 and No. P101/10/1230 and by Centre for research of multiphase flow and thermodynamics processes in renewable sources and energetics - NEW ENERGY, reg. n. CZ.2.16/3.1.00/22130 supported by European Union

\section{References}

1. M. Matejka, P. Doerffer, M. Kurowski, 31. Setkání kat. mech. tekutin a termomech., 149-152, (2012)

2. P. PICK, Diploma Thesis, CTU in Prague, (2007)

3. Z. Broučková, P. Šafařík, Z. Trávníček, Fluid Mech. and Thermo. - Proc. of Student' Work 2010/2011, CTU in Prague, p.23 (2011),

4. V. Skála, Diploma thesis, CTU in Prague, (2011)

5. P. Pick, Dissertation thesis, CTU in Prague, (2014) 